SUBSTANCE OF A LECTURE ON THE

PHYSIOLOGICAL ACTION OF LEAD,

DELIVERED AT THE

BLENHEIM-STREET SCHOOL OF MEDICINE, BY

ROBERT D. THOMSON, M.D., London.

WHEN we pour a solution of acetate of lead in distilled water into Thames water, or when we drop a crystal of acetate of lead into the same liquid, you observe that there is an immediate precipitate, which subsides in the form of white flocks, and as long as the solution continues to afford a precipitate, you cannot detect any acetate of lead in the filtered liquor, at least in an uncombined state; for if you pass a current of sulphuretted hydrogen through the filtered liquid, which you know is the usual method of separating lead from its solutions, you never can obtain lead (if at all) free from animal or vegetable matter. This property which acetate of lead possesses, of presipitating animal and vegetable matter, that is, of forming compounds with them, which are insoluble in water, renders it indispensable in purifying fluids from these bodies, and is peculiarly serviceable in pharmacy, when we wish to obtain the alkaloids in a state of purity. I have also employed it for obtaining approximations to the quantities of animal and vegetable matter's in different kinds of water, so as to be able to exhibit, in some degree, their relative parity.

In India, as in this country, the causes of diseases have been attributed to every possible source, just as the imagination of the speculator brightened up. I recollect that upon one occasion cholera having broke out with great virulence at Bombay, the impure water of the tropics was immediately set down as the cause of it ; the disease, however, was limited in its range, and in the course of a short period it entirely subsided. Now at this very time thousands of persons were in the habit of drinking the same water with impunity, and those who recovered from cholera used the same water before, during, and after their attack, without having any relapse. To prove, however, the fallacy of the opinion in an appreciable manner, I took equal weights of Thames water and the tank-water of Bombay, added to each a solution of acetate of lead, till all precipitation ceased, threw the precipitates on a filter, and washed and dried them; by heating these precipitates strongly the organic matter was drawn off, and the metallic lead remained behind; this, when weighed, was always more considerable in the case of Thames than of Bombay water, from which it was inferred that a greater quantity of organic matter was contained in the former than in the lat. ter, and hence an inference of the erroneous nature of the opinion, which ascribed a greater impurity to Bombay than to Thames water. The latter, in fact, contains more organic matter than any specimen which I have examined, even from the most frequented tanks of India, where the inhabitants are constantly bathing, and stirring up the sediment from the bottom. If Bombay water, therefore, so readily produced cholera, we should expect that Thames water would more powerfully occasion the disease; but all those who have experienced the use of Thames water in long voyages, can bear testimony to its wholesomeness and its exceedingly agreeable taste. It possesses a sweetness which $I$ have never experienced in any other. The great affinity existing between lead and organic matter in solution is of great practical import; for example, it is usual to order a certain number of grains of acetate of lead to be dissolved in water, when it is to be employed as a lotion. Now, if you were to employ water containing organic matter, or carbonic acid, you cannot regulate the strength of your solution without determin. ing first the quantity of these impurities which it contains; if you were unable to procure distilled water, rain water, or spring water, which are pure, in the order named, you might employ Thames water, first adding acetate of lead to it as long as a precipitate fell, and then filtering it through blotting-paper. The filtered liquid might then be employed to dissolve acetate of lead to auy strength required. A similar remark applies to nitrate of silver, which should always be dissolved in distilled water, never in Thames water, unless with the precaution here given, because it is decomposed both by the common salt and by the matter contained in the water.

Now it is to be remarked, that when acetate of lead is added to a liquid containing organic matter, the whole of the lead unites with the organic matter as long as there exists any of the organic matter in the liquid; that is to say, if you were to add three grains of acetate of lead (which we shall suppose requires six grains of organic matter to precipitate it) to a solution containing eight grains of organic matter, the whole of the lead would be thrown down in a solid form, and two grains of organic matter would still remain in solution.

Keeping these actions in view, the following case will be interesting, which occurred at University College Hospital, in December, 1836 :-

CASE.-Sarah Crabbe, a young woman, aged 18 years, was brought to the hospital in a coach, and while she was carried out of the coach she drew a deep sigh, and expired. It appeared from the evidence taken before the ccroner, that she had been in 
low spirits about a love affair, and that she had drunk the same morning a quantity of sugar of lead and alum dissolved in water, which she had bought to do her gown with, and that the same evening she left the house in Drury-lane, where she was residing, and walked to Camden Town, to the house of a nurse, with whom she was acquainted, and there swallowed a pennyworth of nux vo. mica, in the temporary absence of the nurse, having previously purchased the poison of a druggist in the neighbourhood.

Mr. Tayloe, of the hospital, was so good as to send me about a fluid ounce of the contents of the stomach ; the matter was of the consistence of treacle, had a strong smell of acetic acid, and reddened litmus test paper. I readily detected lead, which I now show you, and strychnine, but could obtain no trace of alumina; hence, in a short notice of the case which was published at the time, I remarked, that " all the nlum had been carried away from the stomach, while much of the lead which had been swallowed along with the alum nine hours before death, still remained.

These facts prove that lead, by its combiuation with the vegetable and animal mat. ter in the stomach, becomes a somewhat permanent resident in the viscus. How is it detained? Is it by its combination with the coats of the stomach, or by getting imbedded in the folds of this organ? A rather interesting inquiry also results from these facts, viz, relative to the state in which the lead swallowed in the form of acetate of lead as a remedial agent, exercises its therapeutic powers. Can it be in the form of acetate of lead after that salt is thoroughly acted on by the vegetabie and animal matter?

From these considerations it appeared to be impossible that the idea promulgated by Dr. Anthony Todd Thomson could be cor. rect, viz., that salts of lead only produce death when they are reduced to the state of carbonate. The view which appeared to ne the correct one, was that death would only be occasioned when the quautity of aninal matter decomposed was so great as to produce disorganisation of organised membranes, and that if it could be reduced to the state of oxide without combining with organic matter, it would be carried off without injury to the animal economy.

In a lecture, by Dr. Sigmond, lately printed in THE LANCET, the author states that he differs in opinion from $\mathrm{Dr}$. A. $T$. Thonson, but withont giving us the grounds of his opinions. As he has also omitted any notice of the recer, $t$ important experiments upon the animal structure with lead, and as the subject is one of experimental and not of argumentative investigation, I shall make no apology for presenting you with a summary of the excellent observations of $\mathrm{Dr}$. C. G. Mitscherlich, of Berlin, published in
Miuller's "s Archiv. fut Anatomie" \&c, Heft IV, and V., 1836, which only came into my hauds last year.

1st. Action of Acetale of Lead upon Albu. $m e n, 8:-W$ Wen acetate of lead is added to a solution of white of egg, a precipitate is immediately produced, which dissolves in an excess of albumen, and also by the addl. tion of an acid; filtering the precipitate, the liquid which passes through the filter contains a compound of albumen and lead in solution. Hence there are two con. pounds, at least, formed, one soluble, and the other insoluble, in water. Similar re. sults take place with casein, salivary mat. ter, osmazoin, and the pepsin of Sehwann, while no compounds are formed with fibriu and glue.

2nd. Action of Acetate of Lead upon the Solids and Fluids of the Body.-When a solution of ace tate of lead is poured upon a muscle the latter becomes white, and the whiteness penetrates into the substance of the muscle in proportion to the quantity of acetate of lead; this is a new compound, formed between the matter of the muscle and the acetate of lead; other metallic salts form analogous compounds. When acetate of lead is applied, in sinall doses, to the intestinal canal, it is completely decompose ed by the secretion from the mucous mem. brane, but when it is administered in large doses, the secreted fluid is entirely decom. posed, and the excess of acetate of leud peretrates to the living surface of the mem. brane, and combines with it, and what is termed in common language corrosion takes place. The same result is equally produced on a dead or living mucous surface, or upou the conjunctiva, in wounds, on the skin, on raw surfaces, in cellular tissue, nervous or muscular fibres. When solution of acetate of lead is introduced into the circulation. the water of the blood is precipitated by the metallic salt, - the blood globules are not affected, except that they are no longer com. pletely transparent. The effect upon the blood seems to be the combination of the albumen with the acetate of lead,

3rd. Experiments on Animals.-The l'esult of these experiments was, that when to rabbits half a gramme ( 7 grains) of acetate of lead dissolved in distilled water, was adminis. tered daily, the animal died after $10-12$ doses (77 $\frac{1}{2}-93$ grains), with colresponding alterations of the structure of the internal memblanes. The first doses did not pro. duce much efrect, except that less feculent matter and urine were voided. After the $6-7$ doses the animal became weaker, lay more frequently on its belly, and in some cases was affected with spasms, which subsequently disappeared. The weakness daily increased, the excretions diminished, respiration became oppressed, and death took place, accompanied with episthotonos. On dissection the stomach was found to contain 
a yellowish fuid, which possessed an acid reaction, and in which white insolubje flocks floated. This was similar to the result which I obtained in the case of Sarah Crabbe. I Lead could readily be detected in the filtered fluid, and also in the solid contents. The mucous secretion was converted into a white homogeneous looking mass; in most cases the mucous coat was somewhat, but very slightly altered; a large number of swall white points could be delected on its surface, produced by the chemical action of the acetate of lead upon the structure. The small intestines were healthy, without white points, containing tenacious mucus and very little fluid. The coecum was filled with a pap-like brown mass, but was healthy. The rectum contained a small quantity of hard fæces, which yielded lead by analysis. The blood was considerably charged. The proportion of serum to the solid materials was much diminished, and the latter were strongly coagulated; the serum was thick. The lungs were mostly free from air and blood, and posasessed only some dark points; but in some cases they were much darker than usual, from the black coagulated blood. The bronchial tubes were healthy. The kidneys were healthy; the bladder was generally filled with clear urine; all the other organs were healthy.

When the dose was increased, as when 10 grammes ( $154 \frac{1}{2}$ grains) of acetate of lead were injected at once into the stomach, the efiects produced were more rapid and more aggrovated; the respiration and circulation became very soon increased; the dung was firm, but whiter than usual ; the urine frequently contained white flocks, and sometimes bloody. After some hours the animal became much weaker and the respiration difficult; it lay much on its belly, and was affected by convulsions. The debility gradually increased to such an extent that the head could no longer be sustained but sank between the fore-legs; the pulse became inperceptible, and death took place with opisthotonos; the time which the dose required to produce the fatal effect varied from three to twelve hours. In addition to the appearances detailed as observable on dissection after death from small doses, the slimy-looking mass produced by the decomposition of the mucous secretion, was found to cover the mucous coat to the depth of 1-2 lines; the mucous coat had undergone very considerable alteration : it formed a gray, whitish, dry mass, which resembled very much in consistence coagulated albumen or curd. A similar appearance is produced by digesting mucous membrane in a strong solution of acetate of lead. The muscular coat was also affected, being in some places completely white. The whole of the mucous coat of the small intestines was acted on by the lead, being white, thickened, and dry. In numerous places red spots were observable which were ecchymosed points. The acetate of lead appeared to have penetrated through the cor. roded mucous coat which formed the folds of the intestine until it came in contact with the blood-vessels, which it also corroded; it then mixed with the blood, and deposited the dark coagula. The mucous coat of the blood-vessels was also in many cases acted on by the lead in addition to the coagulation of the blood. The urine was frequently milky and sometimes bloody; in the latter case the outer surface of the kidney was found to be darker than usual.

From these experiments it is evident that when acetate of lead is introduced into the alimentary canal it speedily combines witb the fluid and solid substances with which it comes in contact, and that it never reaches the blood-vessels in the state of acetate of lead. In a therapentical point of view, the question, therefore, for solution is, What is the action of the compounds of acetate of lead and animal matter upon the economy? Before we can prescribe with safety acetate of lead for hamorrhages from the lungs, it is obvious that this question must be solved. Mitscherlich accordingly extended his re. searches to this point.

4th. Action of Acelate of Lead when com. bined with Albumen and Acetic Acid.-Half a gramme (7.75 grains) of sugar of lead was dissolved in water, precipitated by an ex. cess of albumen, and the compound dissolved in acetic acid. This dose was administered to rabbits every other day. Death occurred from the seventh to the sixteenth day. It required $\mathbf{3 . 5}$ grammes (54. grains) of acetate of lead to produce death, or half the quantity which was necessary of pure acetate of lead. The symptoms were similar to the cases detailed. After the fourth dose great weakness was exhibited. The sixth dose produced bloody urine. The seventh dose caused the animal to be very sick. On the sixteenth day violent spasms came on at intervals of four hours, and death took place with opisthotonos. On dissection the mucous membiane of the intestiral canal was found to be uncorroded, of the natural colour in the stomach, but reddened in the small intestines; a number of brown. ish-black points were observable at the large curvature of the stomach. The lungs contained very little air. The hidneys exhibited in some parts of the cortical sub: stance a blackish-brown appearance, similar to what is found when bloody urine has been discharged. The blood-vessels contained very little blood. The blood was strongly coagulated and dark. The serum possessed a cherry-red colour. From these experiments it is obvious that the com. pounds of albumen and acetate of lead, when dissolved in acetic acid, are twice as poi. sonous as when acetate of lead is used alone. 
In the latter case a small portion only of the compounds is dissolved by the free hydrochloric and lactic acid of the stomach, and it is only the soluble compounds which are poisonous.

5th. Action of Acetate of Lead on Wounds. -An incision half an inch in length was made near the spine of a rabbit, and the cellular substance detached by the handle of the scalpel so as to form a bag extending to the mesial line of the abdomen. Two gram. mes (30.86 grains) of acetate of lead were introduced in substance and the edges of the wound brought together. On the following day the same experiment was performed on the opposite side. After two days two grammes (altogether six grammes, or 92.6 grains) were introduced into a wound in the posterior extremities. The animal became lame in these limbs, as dissection showed, from the corrosion of the muscles by the lead. Respiration was affected by the size of the lesion in the pectoral and abdominal muscles, and the animal died on the evening of the following day without strong convulsions. On dissection the parts in the neighbourhood of the wounds were converted into a white, dry, easily divisible mass; the corrosion extended to 1,2 , and 3 lines in depth, and in one place to the peritoneum. The arteries and veins on the corroded surfaces were also white. The blood in the other vessels appeared unchanged.

In another experiment half a goamme combined with albumen, and introduced under the skin, produced death in twenty hours, with symptoms of opisthotonos ; and in a third trial, one gramme (15.43 grains) of acetate of lead, dissolved in acetic acid, produced death in three hours. Hence it would appear that pure acetate of learl corrodes chemically, and can only produce symptoms of general poisoning when it comes in contact with surfaces discharging free acid which may dissolve the compounds. In the other cases the symptoms were similar to those induced by the introduction of the same substances into the stomach.

6th. Examination of the Blood and Urine for Lead.-Mitscherlich obtained a very minute quantity of a black precipitate by means of sulphuretted hydrogen, which he suspected to be sulphuret of lead, but the quantity was too minute to decide. No lead was found in the urine.

Conclusions.--From the experiments which have been detailed we learn,-

1st. That when acetate of lead is applied directly to the stomach, it acts upon the mucous secretion, and if the dose is sufficient, upon the mucous coat itself, corroding it, according to the usual term of expression, or, more correctly, forming a chemical combination with the matter of which it consists. According to the strength of the dose the mucous coat of the whole intestinal canal is more or less affected. Hence if we wish to administer acetate of lead to stop hamor. rhage of the intestines, it should be given in solution in water.

2nd. Acetate of lead only produces symp. toms of general poisoning when the compounds which it forms with organic matter are in solution in acids. Hence the method recommended by Dr. A. T. Thomson, of giving acetate of lead with jinegar, is the very method for increasing the poisonous effects of acetate of lead; the occurrence of carbonate or oxide of lead in the stomach in a free state is impossible after the adminis. tration of acetate of lead.

3rd. These experiments elucidate the ac. tion of acetate of lead when employed to overcome inflammation of mucous mem. branes. It first decomposes the secretion, corrodes the membrane, penetrates to the blood-vessels, which it also corrodes, coangulates the blood; it, in fact, kills the part, which ultimately falls off either by detach. ment, or by beiug dissolved.

These experiments are of great value in a therapeutical point of view, because they afford a kind of type of the action of all metallic salts ; the nature of their therapeu. tic power being strictly an object of chenical research. The presence of lead in the blood, and its state of combination in that fluid still remains to be determined.

UNIVERSITY COLLEGE HOSPITAL.

\section{ANIMAL MAGNETISM.}

ON Thus soay, May 10th, a remarkable exhibition of the effects of " animal magnetism" took place at this hospital, at which nearly 200 medical gentlemen, students, and other observers were present. Among the company were the Marquis of Anglesea, Sir Charles Paget, the Earl of Burlington, the Duke of Roxburgh, Earl Wilton, Iord Dinorben, Dr. Faraday, Sir Jos. De Courcy Laffan, Sir J.South, and several Members of Parliament. The proceedings were conducted in the theatre of the hospital, by Dr. Elliotson, assisted by Mr. Wood. The company, so many of them as could find sitting-room, being seated in the area of the theatre after the benches above were filled, Dr. Elliotson presented on the floor a girl named Okey, eighteen years old, somewhat chlorotic in countenance, and diminutive for her age. She was brought from the wards of the hospital, where she had been placęd under treatment for epilepsy of many months duration. The girl had several times before, as we were informed, been made the subject of public experiments in magnetism. Her figure was sumewhat womanish, but her face and head were those of a child. No particular character was indicated by her features, or by any expression of feeling that could be observed in them. 